\title{
Effect of diets with different fat contents on the development of diabetes in female Zucker diabetic fatty rat with leptin mutation
}

\author{
Renata Köhlerová1, Martina Sznapková2 ${ }^{1}$, Rastislav Slavkovský ${ }^{3}$,Alena Jiroutová ${ }^{1}$ \\ ${ }^{1}$ Charles University in Prague, Faculty of Medicine in Hradec Králové, Department of Medical Biochemistry, \\ Czech Republic \\ ${ }^{2}$ University of Pardubice, Faculty of Chemical Technology, Department of Biological and Biochemical \\ Sciences, Czech Republic, \\ ${ }^{3}$ Contipro Biotech, Laboratory of Cell Physiology, Dolní Dobrouč, Czech Republic
}

Received November 5, 2012

Accepted June 19, 2013

\begin{abstract}
The aim of the study was to develop a diet which causes stable hyperglycaemia and development of diabetes in female Zucker diabetic fatty (ZDF) rats. We also examined whether worsened wound healing is caused only by hyperglycaemia or whether it is caused by more factors. Four types of special diets with a different content of fat were fed to eight groups of 3-7 $(f a / f a$ or $f a /+)$ rats. The following diets were used: H1 (24.6\% fat), H2 (33.2\%), C13004 (25.6\%), and St1 (3.4\%). We detected significant diet-dependent changes of weight and concentration of glucose in animals with leptin mutation $(f a / f a)$. All examined indicators were significantly $(P<0.001)$ higher in $(f a / f a)$ animals compared to the $f a /+$ ones no matter what diet they ate. All diets with high-fat content caused increased glycaemia, but only the diet with $24.6 \%$ fat caused a significant $(P<0.01)$ increase of glycaemia. Our results have proved that this diet is the most suitable to invoke and keep hyperglycaemia. The diet with $25.6 \%$ fat is suitable to invoke stable slightly increased glycaemia $(10 \mathrm{mmol} / \mathrm{l})$ and hyperinsulinaemia. On the other hand, the diet with $33.2 \%$ fat is unsuitable. We did not observe a significant influence of diet on wound healing. We developed a new diet more suitable for induction of stable hyperglycaemia in female ZDF rats than commercially available mixtures. Our study is the first to present recommendations for adjusting a high-fat diet to produce stable hyperglycaemia and hyperinsulinaemia in the rat model.
\end{abstract}

Leptin mutation, glycaemia, insulinaemia, MMP-3, wound healing

Type 2 (DM2T) diabetes mellitus (DM) is the most common metabolic disease that arises due to a relative lack of insulin. The cells are not able to intake glucose which results in hyperglycaemia. Long-term hyperglycaemia leads to serious complications including retarded wound healing. Reliable animal models are necessary to observe the disease development and to test suitable medication (Bartosikova et al. 2003; Lukacinova et al. 2008).

Zucker diabetic fatty (ZDF) rats can be used as a suitable model (Slavkovsky et al. 2011). Rats with $f a / f a$ genotype develop genetically dependent obesity and subsequently diabetes due to a point missense mutation in extracellular domain of the leptin receptor. It is characterized by non-insulin dependent DM accompanied by hyperglycaemia, neuropathies, nephropathies, insulin resistance, mild hypertension, hypertriglyceridaemia, hypercholesterolaemia, polyphagia, polyuria, polydipsia, protein glycation, and microvascular damage. The development of diabetes in these animals resembles the situation seen in humans: animals gradually evolve from hyperinsulin-euglycaemic state to hyperglycaemic state with relative insulin deficiency (Finegood et al. 2001; Leonard et al. 2005; and Slavkovsky et al. 2011).

However, to develop DM due to over nutrition, these rats must be fed on high-energy, high-fat diet. Originally, the rats' provider Charles River Laboratories International, Inc. 
recommended the $\mathrm{C} 13004$ diet. In the year 2007 the composition of the $\mathrm{C} 13004$ diet had been changed. Our results concerning hyperglycaemia induction by this newly composed diet did not correspond either to the values given by the provider or to the values published with original C13004 composition (Corsetti et al. 2000). The company itself later admitted that the diet was not efficient. While the commercially available diet with $4.5 \%$ content of fat (Purina 5001) is enough to develop diabetes in male rats with leptin mutation, the situation in female rats is more complicated.

The aim of our study was to develop new diet for rats and compare its effect on development of diabetes mellitus with the standard laboratory diet and the current high fat laboratory C13004 diet.

Animals

\section{Materials and Methods}

The experimental protocol was approved by the Animal Welfare Committee of Charles University in Prague, Faculty of Medicine in Hradec Králové (No. 12420/2011-30). In the experiment we used 40 female Zucker diabetic fatty rats that originated from the Charles River Laboratories International, Inc. (USA) and were purchased from Anlab (Prague, Czech Republic). The offspring were bred in the vivarium at the Faculty of Medicine in Hradec Králové, Charles University by mating non-obese heterozygous $(\mathrm{fa} /+)$ carrier parents or by mating $f a / f a$ obese males with $f a /+$ females. The rats were kept under standard conditions. They received food and drinking water ad libitum.

Leptin mutation genotypization

All rats were genotyped using a modified PCR-RFLP method. DNA was isolated from a piece of the tail or the phalanx obtained at the age of 3 weeks using a DNeasy Blood and Tissue Mini Kit (Qiagen, Hilden, Germany). The methods were described in detail by Slavkovsky et al. (2011).

Types of diets

Rats at the age of 4 weeks were divided into eight groups of 3-7 animals. There were 4 groups of $f a / f a$ and 4 groups of $f a /+$ rats. According to type of diet (different amount of fat), there were following groups: H1 (24.6\% fat), H2 (33.2\%), C13004 (25.6\%) and St1 (3.4\%); each for $f a / f a$ and $f a /+$ rats. The St1 is standard laboratory diet (ST-1, VELAS, a.s., Lysá nad Labem, Czech Republic) and C13004 is commercial high fat laboratory diet (Research Diets, New Brunswick, NJ, USA). The other diets H1 and H2 (Table 1) were based on mixture of St1, pork fat, corn starch (maizena), and sugar. The ingredients were mixed together and water was added as necessary. The final mixture was shaped into loaves and dried for $48 \mathrm{~h}$ in an oven heated to $60{ }^{\circ} \mathrm{C}$.

Table 1. Composition of diets used for feeding Zucker diabetic fatty rat in our experiment.

\begin{tabular}{lcccc}
\hline Composition & H1 (\%) & H2 (\%) & C13004 (\%) & St1 (\%) \\
\hline Proteins & 14.4 & 12.0 & 14.6 & 24.0 \\
Carbohydrates & 44.8 & 39.8 & 46.6 & 50.5 \\
Fats & 24.6 & 33.2 & 25.6 & 3.4 \\
Fibrous material & 2.2 & 1.9 & 1.4 & 4.4 \\
Ash & 3.9 & 3.3 & 4.9 & 6.8 \\
Moisture & 10.1 & 9.9 & 7.8 & 12.5 \\
\hline
\end{tabular}

Diets C1304 and St1 are commercial, diets H1 and H2 are self-prepared, St1 is a low fat diet.

Weights of rats were regularly measured every two weeks since the age of 4 weeks from birth (week 0 ); i.e. $6 \times$ during the 12 -week period.

A portable glucose meter, AccuChek-Go (Roche, Basel, Switzerland), was used for glucose measurement using a drop of blood from the tail vein every two weeks, same as the measuring of weight. The rats were not anaesthetized during glucose measurement because anaesthesia could increase glycaemia (Saha et al. 2005).

Measurements of insulin concentration

Blood was collected from the orbital plexus of rats. Heparinized blood was centrifuged and plasma was stored at $-70^{\circ} \mathrm{C}$ until it was analysed. Insulin was measured using the Rat Insulin ELISA kit (H-type, Shibayagi, Japan) 
according to the manufacturer's instructions. Concentration of insulin in unknown samples was calculated using the calibration curve of insulin standards $\left(\mathrm{y}=0.0341 \mathrm{x}+0.0393, \mathrm{R}^{2}=0.9995\right)$.

Wound induction and evaluation

Wounds in rats were induced at the age of 16-18 weeks as described by Slavkovský (2011). Briefly: A fullthickness excision circular skin wound of $2 \mathrm{~cm}$ in diameter was made on the back. Wounds were allowed to heal naturally. The wounds were photographed (EOS D350, Canon, Tokyo, Japan) immediately after induction (Day 0) and then every second or third day. The wound area was measured using Image J software (NIH) calibrated to standard length using a millimetre ruler. The relative wound size was expressed according to the following formula: \% wound area $=$ wound area on Day n/wound area on Day 0. The animals were sacrificed on Day 10 and their granulation tissue (GT) was collected at the same time. The sample was stored in RNA stabilizing solution (Qiagen) and frozen to $-70{ }^{\circ} \mathrm{C}$.

Evaluation of MMP-3 expression in the granulation tissue

RNA was isolated from the GT sample (RNeasy Lipid Tissue Mini Kit, RNeasy Fibrous Tissue Mini Kit Qiagen). The amount of RNA was measured using spectrophotometer while the quality was detected using electrophoresis. Total RNA (1 mg) was transcribed to cDNA using the cDNA archive kit (Applied Biosystems, Foster City, California). Synthesized cDNA (High-Capacity cDNA Reverse Transcription Kit, Applied Biosystems) was used to study MMP-3 gene expression using rt RT-PCR method. Glyceraldehyde 3-phosphate dehydrogenase (GAPDH) rat gene (TaqMan Gene Expression Assays Applied Biosystems) was used as housekeeping gen (endogenous control). The results were analyzed using the 7500 Fast System SDS Software and then processed in Microsoft Excel 2007.

\section{Statistical analysis}

Statistics were calculated using the STATISTICA 10.0 data analysis software (StatSoft ČR, s. r. o., 2011). The differences between the control and experimental group were evaluated by Mann-Whitney U test. Differences within the groups were evaluated by Kruskal-Wallis test $(P<0.001$ or $P<0.05)$.

\section{Results}

Weight gain

The weights of $f a / f a$ and $f a /+$ animals were approximately the same in week 0 (Fig. 1). The weight of both groups grew during the time, but weight of $f a / f a$ animals grew faster.

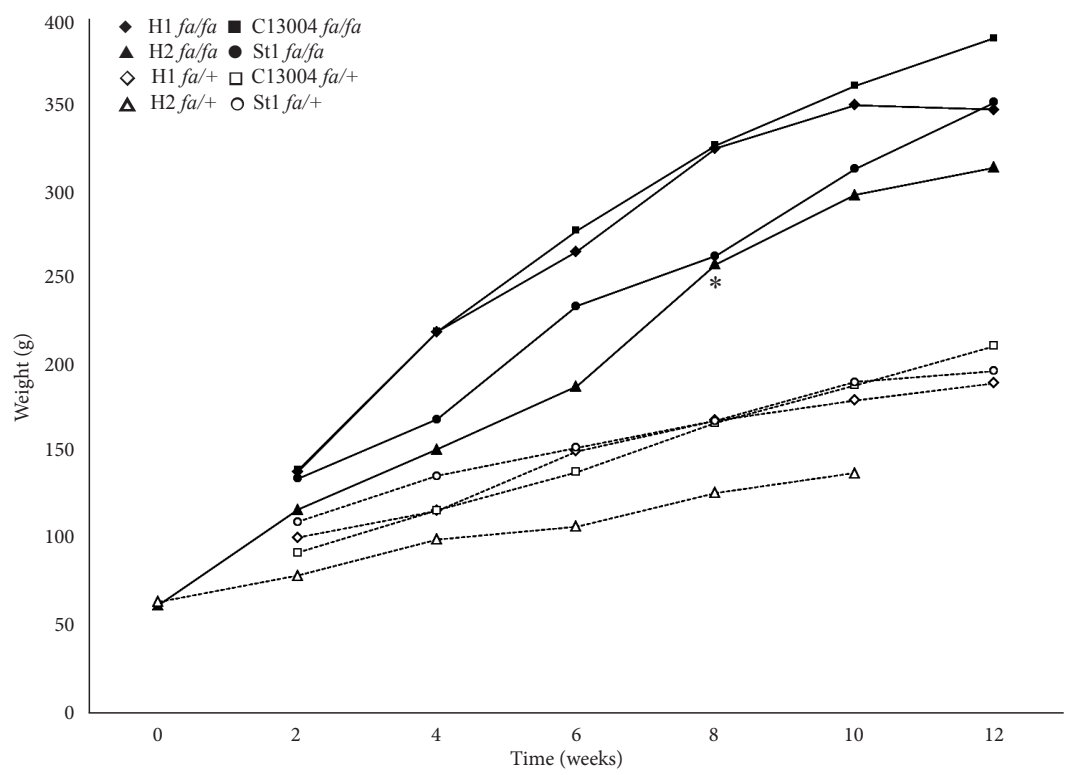

Fig. 1. Influence of different diets on the body weight development in rats

* value is significantly higher in comparison to healthy animals for respective group in the week $8(P<0.001)$ Diets C1304 (25.6\% fat) and St1 (3.4\%) are commercial, diets H1(24.6\%) and H2 (33.2\%) are self-prepared. 
The average weight of $f a / f a$ was significantly higher compared to $f a /+$ animals in week 8 $(P<0.001)$. In week 16 the resulting weight of $f a / f a$ rats was about twice as high as the weight of $f a /+$ controls. Significant differences between diet groups were observed too $(P$ $<0.05)$. Both groups of animals fed high fat diet (H2) showed the lowest weight gain and also high mortality ( $50 \%$ for $f a / f a$ and $71.4 \%$ for $f a /+$ ).

\section{Glycaemia}

Glucose concentrations are shown in Fig. 2. St1 diet had no effect on glycaemia in $f a / f a$ animals, $f a /+$ rats of all diet groups had normoglycaemia. The $\mathrm{H} 1$ and $\mathrm{C} 13004$ diets caused significantly $(P<0.05)$ higher glycaemia in $f a / f a$ animals than St1 as early as after 8 weeks on the diet. Higher glycaemia was detected after 12 weeks in the $f a / f a$ animals fed all three high fat diets (H1, H2, C13004) compared to the $f a / f a$ animals fed St1, but only after using $\mathrm{H} 1$ and $\mathrm{C} 13004$ did this increase become significant $(P<0.05)$. The highest increase in glycaemia was observed in $f a / f a$ animals fed $\mathrm{H} 1$ diet $(26.8 \mathrm{mmol} / \mathrm{l})$, and was higher than hyperglycaemia induced by C13004 (10.4 mmol/l).

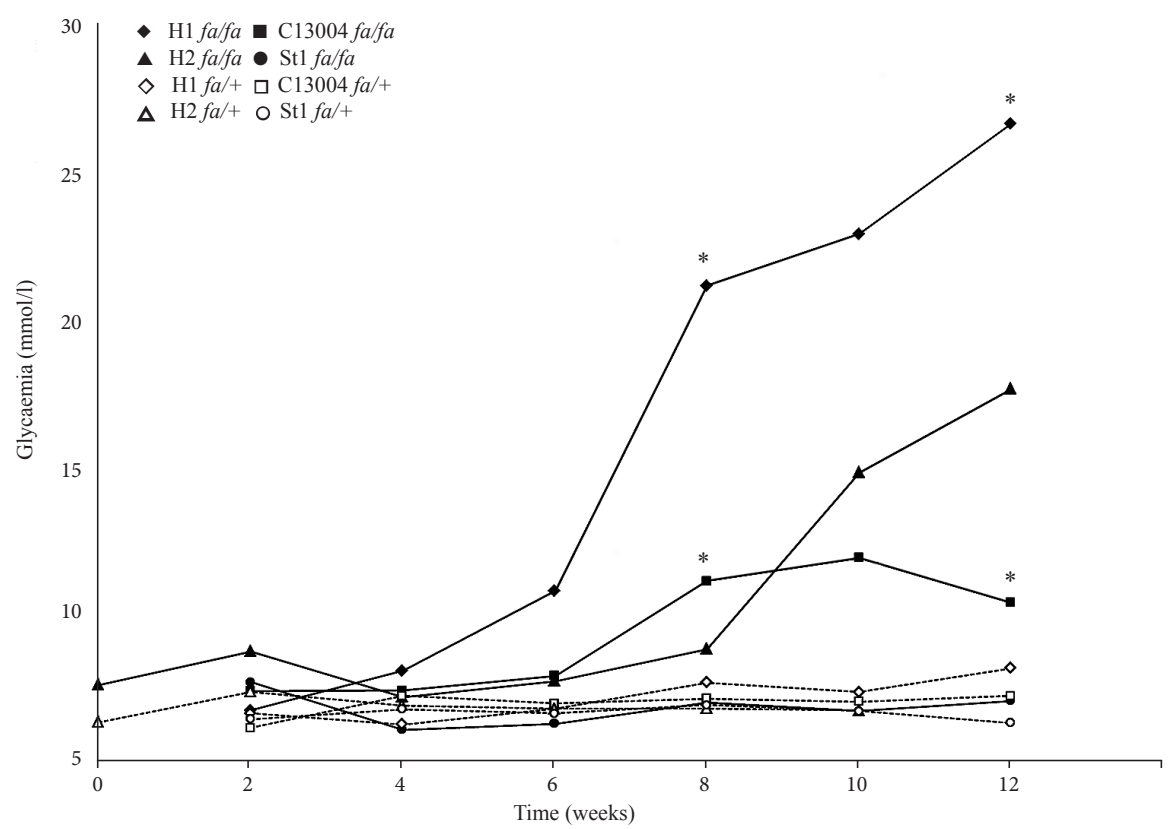

Fig. 2. Influence of different diets in rats on the development of glycaemia

* value is significantly higher in comparison to St1 diet for fa/fa rat $(P<0.05)$. Diets $\mathrm{C} 1304(25.6 \%$ fat $)$ and St1 $(3.4 \%)$ are commercial, diets $\mathrm{H} 1(24.6 \%)$ and $\mathrm{H} 2(33.2 \%)$ are self-prepared.

\section{Insulinaemia}

Levels of insulinaemia were measured before the induction of the skin wound. Concentrations of insulin in $\mathrm{fa} /+$ animals were low and, regardless of the type of diet, did not exceed $2.22 \mu \mathrm{g} / \mathrm{l}$. Nevertheless, we observed significant differences $(P<0.001)$ among the $f a /+$ animals depending on the implemented diet (Fig. 3A).

The levels of insulinaemia between $f a / f a$ animals were significantly $(P<0.001)$ higher with much higher variance (Fig. 3B). Higher concentrations of plasmatic insulin were 
A

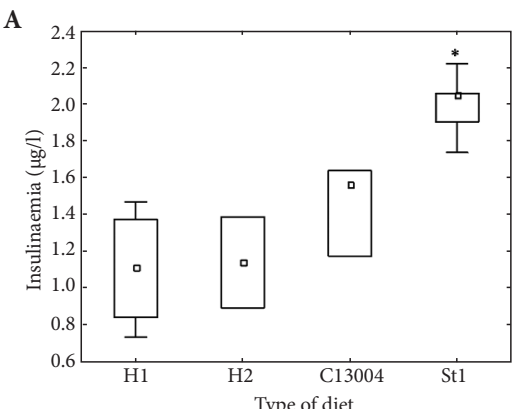

C

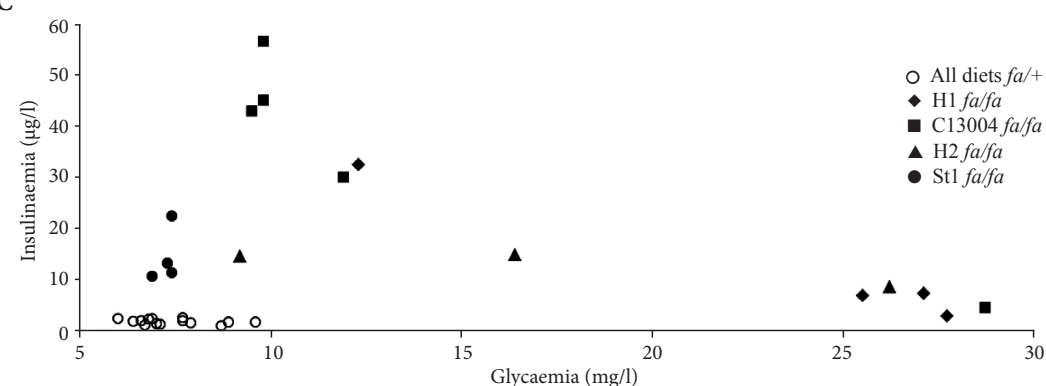

B

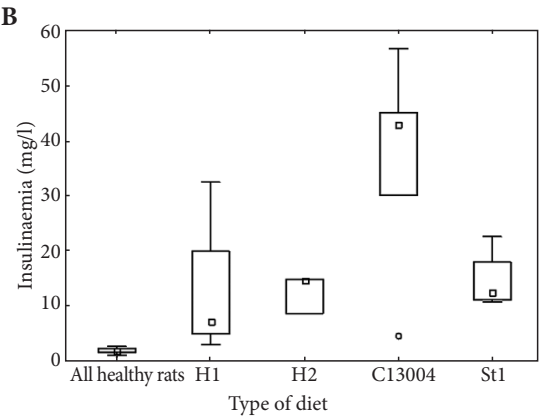

Fig. 3. Values of insulinaemia in rats fed different diets

A - values of insulinaemia in $f a /+$ rats, $\square-$ median, $\square-25 \%-75 \%$, I - range of non-remote values, *value is significantly higher in comparison to other rat groups $(P<0.001)$, B - values of insulinaemia in $f a / f a$ rats, $\square-$ median, $\square-25 \%-75 \%$, I - range of non-remote values, $\circ$ - remote value, C - insulinaemia in dependence on glycaemia

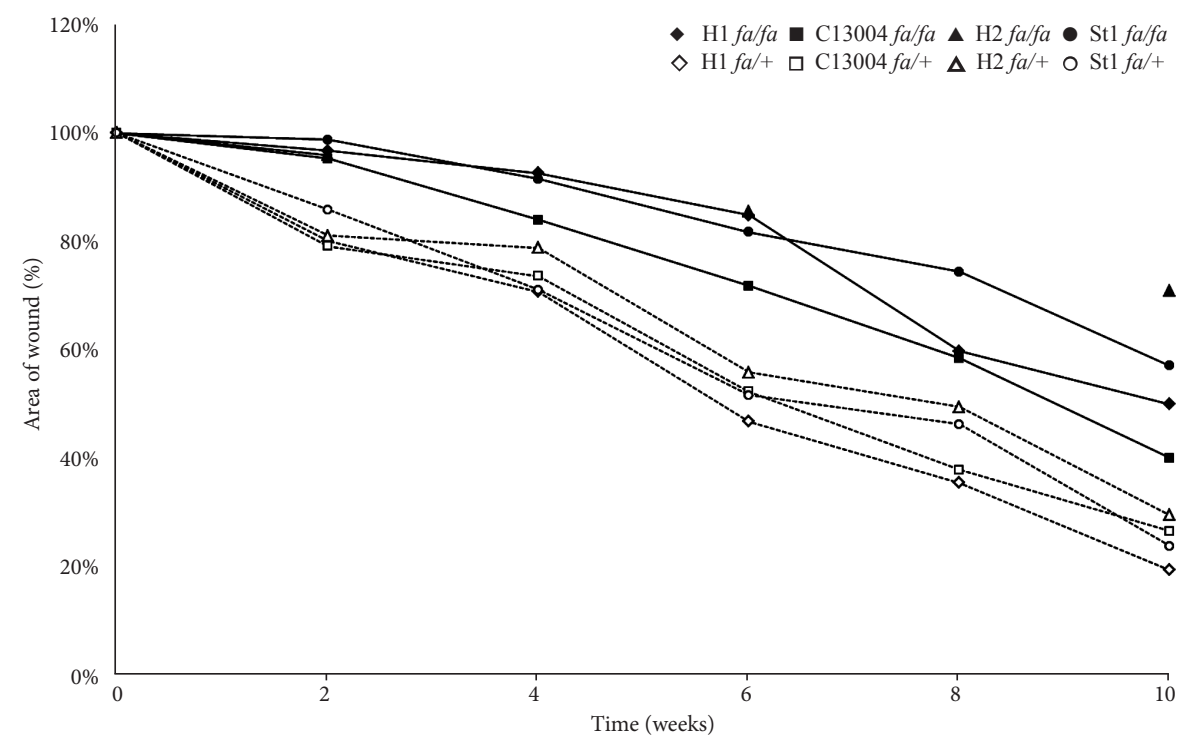

Fig. 4. Average wound area in different groups of rats during time

Diets C1304 (25.6\% fat) and St1 (3.4\%) are commercial, diets H1 (24.6\%) and H2 (33.2\%) are self-prepared. 
detected in the animals fed the $\mathrm{C} 13004$ diet, but the difference was not significant due to high variance.

The relation between concentrations of glycaemia and insulin is shown in Fig. 3C. Both values were normal in $f a /+$ rats. The $f a / f a$ rats fed the standard St 1 diet with $3.4 \%$ of fat had normoglycaemia and $10 \times$ higher concentrations of insulin than $\mathrm{fa} /+$ rats. The rats fed the commercial C13004 diet (25.6\% of fat) had high concentrations of insulin and glycemia around $10 \mathrm{mmol} / \mathrm{l}$. On the other hand, the rats fed the prepared $\mathrm{H} 1$ diet $(24.6 \%$ of fat) had low concentrations of insulin and hyperglycaemia (mean $23.2 \mathrm{mmol} / \mathrm{l}$ ). No trends were observed in animals fed the diet with $33.2 \%$ of fat; these animals responded individually.

\section{Wound closure}

Skin wound contraction was decreased in all $f a / f a$ rats compared to $f a /+$ ones $(P<0.001)$ regardless of diet and glucose or insulin concentrations (Fig. 4). The wound in $f a /+$ animals started to contract immediately after induction, whereas the wound in diabetic animals did not reduce or even grew, and the wound reducing started as late as on day 4. The wound reduced by more than two thirds within 10 days in $f a /+$ rats but only by less than a half in diabetic ones. We observed the slowest wound contraction in animals fed the $\mathrm{H} 2$ diet no matter whether they were $f a /+$ or $f a / f a$. We observed the fastest wound closure in animals fed the C13004 diet within the $f a / f a$ animals.

\section{Expression of MMP-3}

The $f a / f a$ rats had significantly $(P<0.001)$ higher values of MMP-3 expression compared to the $f a /+$ rats (Fig. 5). When we evaluated the influence of the diet, we found significant $(P<0.05)$ difference between $f a / f a$ and $f a /+$ rats fed the St 1 and $\mathrm{H} 1$ diets. The differences between $f a /+$ rats fed different diets were not significant, nor were the differences within the $f a / f a$ group.

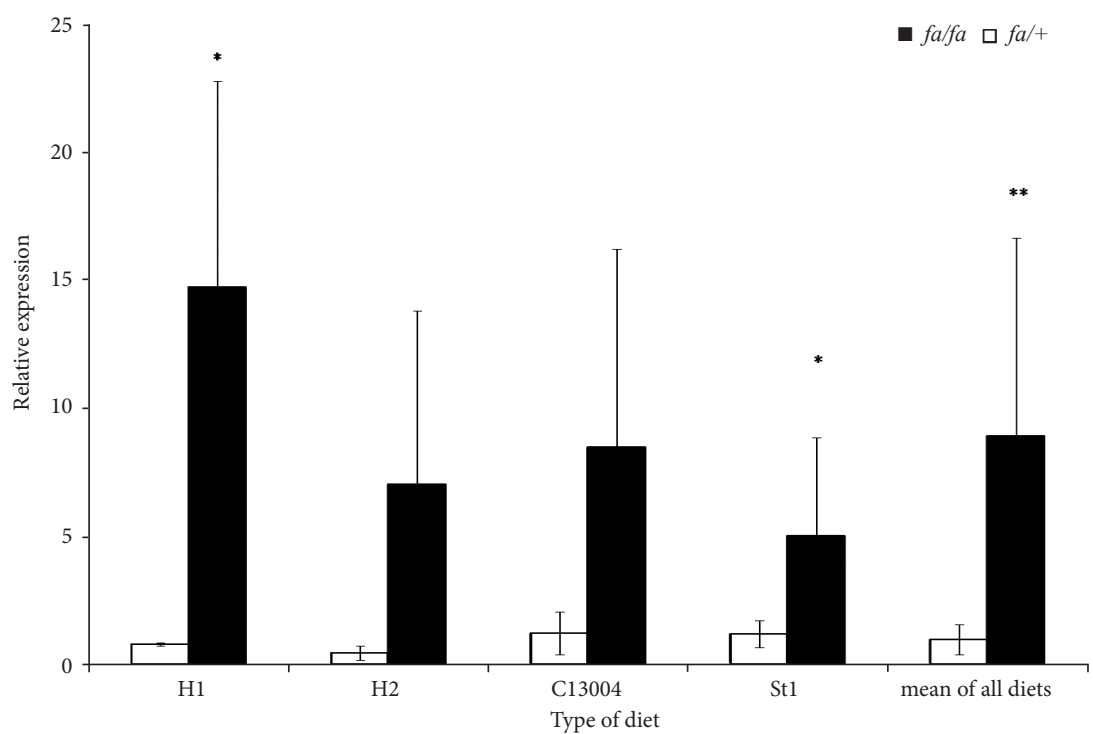

Fig. 5. Matrix methaloproteinase-3 gene expression in granulation tissue of wound on day 10

*value is significantly higher in comparison to fa/ + rat $(P<0.05),{ }^{* *}$ value is significantly higher in comparison to fa/ + rat $(P<0.001)$ Diets C1304 $(25.6 \%$ fat $)$ and St1 $(3.4 \%)$ are commercial, diets H1 $(24.6 \%)$ and H2 $(33.2$ $\%)$ are self-prepared. 


\section{Discussion}

The $f a / f a$ rats increased their weight much faster than the $f a /+$ ones regardless of the fat amount in the diet. The same result was described by Finegood et al. (2001). Rats fed the diet with the highest content of fat increased their weight most slowly in both groups and their mortality was high. This diet seems to be unsuitable for experiments.

Studies concerning the development of diabetes mellitus are usually focused on changes of glycaemia. Corsetti et al. (2000) proved that DM2T was developed in $f a / f a$ ZDF male rats even when being fed a low-fat diet (4.5\%, Purina 5001). On the contrary, in female rats diabetes was developed only when being fed a diet with the content of fat of at least $25.6 \%(\mathrm{C} 13004)$.

Corsetti (2000) observed slightly increased glycaemia at the age of 15 weeks in the $\mathrm{fa} /$ $\mathrm{fa}$ female ZDF rats fed a diet with the fat content of 11\% (Purina 5015 from Purina Lab Diets). Female rats fed low-fat diets had high levels of insulin. This finding does not agree with our observations. We observed only small increase of insulinaemia.

In our study the increase of glycaemia caused by the C13004 diet was not as high as in the study by Corsetti (2000). This diet was recommended as suitable for development of hyperglycaemia by the Charles River Laboratories, USA, but the company itself later recommended the use of the new 12468 diet developed in cooperation with the Purina Lab Diets company. The H1 diet with the same fat content as C13004 is more suitable for development of hyperglycaemias.

Two diets with a similar content and composition of fat influence $f a / f a$ ZDF female rats in different way. The $\mathrm{H} 1$ diet causes high concentration of glucose and low insulinaemia whereas the C13004 diet causes hyperinsulinaemia and slightly higher glycaemia.

The female ZDF rats fed H1 can be used as an important animal model of DM2T in women. The relations between concentrations of glycaemia and insulin are interesting. Both values are low in $f a /+$ rats. The female $f a / f a$ rats fed the standard St 1 diet (3.4\% of fat) had normoglycaemia and $10 \times$ higher concentrations of insulin than $\mathrm{fa} /+$ rats. Female rats fed the commercial C13004 diet $(25.6 \%)$ had high concentrations of insulin and glycaemia of about $10 \mathrm{mmol} / \mathrm{l}$; in contrast, rats fed the prepared $\mathrm{H} 1$ diet had low concentrations of insulin and hyperglycaemia.

Impaired wound healing in diabetic patients is supposedly related to the uncompensated hyperglycaemia. Experimentally this was evaluated by O'Sullivan at al. (2011), who studied healing of incision wounds in Sprague-Dawley rat with streptozotocin-induced diabetes. They observed significantly better healing in rats supported by insulin. On the enzymatic level, impaired wound healing in diabetic terrain is associated with increased activity of tissue matrix metalloproteases (MMPs, Burrow et al. 2007; Guo and DiPietro, 2010) and a decreased effect of MMP inhibitors - TIMPs (Lobmann at al. 2002). In our study the wound healing of $f a / f a$ rats was significantly impaired, but the severity of the impairment did not reflect the value of glycaemia or insulinaemia. Therefore we presumed that worsened healing observed in diabetic $f a / f a$ rats could be related to changes in MMPs.

MMP-3 (stromelysin-1) cleavages especially non-collagen compounds of ECM and it plays an important role in wound contraction (Bullard et al. 1999) and angiogenesis (Zheng et al. 2007). We decided to use MMP-3 because the highest differences in gene expression were observed between $f a / f a$ and $f a /+$ rats (Slavkovsky et al. 2011). We found in our study that MMP-3 expression in granulation tissue was higher in $f a / f a$ female rats, whose wounds healed worse. These results correspond well with the findings of Kilpadi et al. (2006) who found that decreased MMP-3 and MMP-9 levels during therapy of pressure ulcers correlate with positive wound healing outcomes.

Diet composition can significantly influence concentrations of glucose and insulin in $\mathrm{fa} /$ $\mathrm{fa}$ female rats. The $\mathrm{H} 1 \mathrm{diet}$ is suitable to develop and keep hyperglycaemia. The C13004 
diet is suitable to develop hyperinsulinaemia and a stable, slightly increased glycaemia $(10 \mathrm{mmol} / \mathrm{l})$. Our results suggest that correction of glucose and insulin concentrations into physiological range is not sufficient enough to influence all undesirable symptoms of DM. The wounds in $f a / f a$ rats healed slowly regardless of weight, glycaemia, and insulinaemia. Impaired healing was accompanied by increased expression of MMP-3 in $\mathrm{fa} / \mathrm{fa}$ rats in comparison to fa/+ rats.

\section{Acknowledgements}

Authors wish to express their thanks to Mgr. Petr Čoupek for his help with the statistical analysis and to Dr. Stanislav Mičuda for the offer of the real-time PCR instrument. This research was supported by the programme PRVOUK P37/01.

\section{References}

Bartosikova L, Necas J, Suchy V, Kubinova R, Vesela D, Benes L, Bartosik T, Illek J, Salplachta J, Klusakova J, Bartosova L, Strnadova V, Frana P, Franova J 2003: Monitoring of antioxidative effect of morine in alloxaninduced diabetes mellitus in the laboratory rat. Acta Vet Brno 72: 191-200

Bullard KM, Mudgett J, Scheuenstuhl H, Hunt TK, Banda MJ 1999: Stromelysin-1-deficient fibroblasts display impaired contraction in vitro. J Surg Res 84: 31-34

Burrow JW, Koch JA, Chuang HH, Zhong W, Dean DD, Sylvia VL 2007: Nitric oxide donors selectively reduce the expression of matrix metalloproteinases- 8 and -9 by human diabetic skin fibroblasts. J Surg Res 140: 90-98

Corsetti JP, Sparks JD, Peterson RG. Smith RL, Sparks CE 2000: Effect of dietary fat on the development of non-insulin dependent diabetes mellitus in obese Zucker diabetic fatty male and female rats. Atherosclerosis 148: $231-241$

Finegood DT, McArthur MD, Kojwang D, Thomas MJ, Topp BG, Leonard T, Buckingham RE 2001: Beta-cell mass dynamics in Zucker diabetic fatty rats. Rosiglitazone prevents the rise in net cell death. Diabetes 50: 1021-1029

Guo S, DiPietro LA 2010: Factors affecting wound healing. J Dent Res 89: 219-229

Kilpadi DV, Stechmiller JK, Cowan L, Kieswetter K, Schultz G, Bellis SL 2006: Composition of wound fluid from pressure ulcers treated with negative pressure wound therapy using V.A.C. therapy in home health or extended care patients: A pilot study. Wounds 18: 119-126

Leonard BL, Watson RN, Loomes KM, Phillips AR, Cooper GJ 2005: Insulin resistance in the Zucker diabetic fatty rat: a metabolic characterisation of obese and lean phenotypes. Acta Diabetol 42: 162-170

Lobmann R, Ambrosch A, Schultz G, Waldman K, Schiweck S, Lehnert H 2002: Expression of matrixmetalloproteinases and their inhibitors in the wounds of diabetic and non-diabetic patients. Diabetologia 45: 1011-1016

Lukacinova A, Mojzis J, Benacka R, Keller J, Maguth T, Kurila P, Vaško L, Rácz O, Ništiar F 2008: Preventive effects of flavonoids on alloxan-induced diabetes mellitus in rats. Acta Vet Brno 77: 175-182

O’Sullivan JB, Hanson R, Chan F, Bouchier-Hayes DJ 2011: Tight glycaemic control is a key factor in wound healing enhancement strategies in an experimental diabetes mellitus model. Ir J Med Sci 180: 229-236

Saha JK, Xia J, Grondin JM, Engle SK, Jakubowski JA 2005: Acute hyperglycemia induced by ketamine/xylazine anesthesia in rats: mechanisms and implications for preclinical models. Exp Biol Med 230: 777-784

Slavkovsky R, Kohlerova R, Tkacova V, Jiroutova A, Tahmazoglu B, Velebny V, Rezacova M, Sobotka L, Kanta J 2011: Zucker diabetic fatty rat: A new model of impaired cutaneous wound repair with type II diabetes mellitus and obesity. Wound Rep Reg 19: 515-525

Zheng L, Amano K, Iohara K, Ito M, Imabayashi K, Into T, Matsushita K, Nakamura H, Nakashima M 2009: Matrix metalloproteinase-3 accelerates wound healing following dental pulp injury. Am J Pathol 175: $1905-1914$ 